\title{
Schisandra chinensis and Morus alba Synergistically Inhibit In Vivo Thrombus Formation and Platelet Aggregation by Impairing the Glycoprotein VI Pathway
}

\author{
Dong-Seon Kim, ${ }^{1}$ Muhammad Irfan, ${ }^{2}$ Yoon-Young Sung, ${ }^{1}$ Seung Hyung Kim, \\ Sun Haeng Park, ${ }^{1}$ Young Hyun Choi, ${ }^{4}$ Man Hee Rhee, ${ }^{2}$ and Ho Kyoung Kim ${ }^{1}$ \\ ${ }^{1}$ Korea Institute of Oriental Medicine, Daejeon 34054, Republic of Korea \\ ${ }^{2}$ Laboratory of Veterinary Physiology and Cell Signaling, College of Veterinary Medicine, \\ Kyungpook National University, Daegu 41566, Republic of Korea \\ ${ }^{3}$ Institute of Traditional Medicine \& Bioscience, Daejeon University, Daejeon 34520, Republic of Korea \\ ${ }^{4}$ Department of Biochemistry, Dongeui University College of Korean Medicine, Busan 614-052, Republic of Korea
}

Correspondence should be addressed to Man Hee Rhee; rheemh@knu.ac.kr and Ho Kyoung Kim; hkkim@kiom.re.kr

Received 14 October 2016; Revised 13 December 2016; Accepted 29 December 2016; Published 18 January 2017

Academic Editor: Victor Kuete

Copyright (C) 2017 Dong-Seon Kim et al. This is an open access article distributed under the Creative Commons Attribution License, which permits unrestricted use, distribution, and reproduction in any medium, provided the original work is properly cited.

\begin{abstract}
Morus alba L. (MAL) extract has been used in traditional medicine for its cardioprotective and antiplatelet effects, while another herbal remedy, Schisandra chinensis (SCC), has been reported to have anti-inflammatory and antioxidant properties. We evaluated underlying cellular changes exerted by extracts of these plants on platelet function and effects of SCC + MAL on in vivo thrombus formation using AV shunt and tail thrombosis-length models in rats. In vitro platelet aggregation, granule secretion, and $\left[\mathrm{Ca}^{2+}\right]_{i}$ release assays were carried out. The activation of integrin $\alpha_{\mathrm{II}} \beta_{3}$ and phosphorylation of downstream signaling molecules, including MAPK and Akt, were investigated using cytometry and immunoblotting, respectively. Scanning electron microscopy (SEM) was used to evaluate changes in platelet shape and HPLC analysis was carried out to identify the marker compounds in SCC + MAL mixture. In vivo thrombus weight and average length of tail thrombosis were significantly decreased by SCC + MAL. In vitro platelet aggregation, granule secretion, $\left[\mathrm{Ca}^{2+}\right]_{i}$ release, and integrin $\alpha_{\mathrm{IIb}} \beta_{3}$ activation were notably inhibited. SCC + MAL markedly reduced the phosphorylation of MAPK pathway factors along with Akt. HPLC analysis identified four marker compounds: isoquercitrin, astragalin, schizandrol A, and gomisin A. The extracts exerted remarkable synergistic effects as natural antithrombotic and antiplatelet agent and a potent drug candidate for treating cardiovascular diseases.
\end{abstract}

\section{Introduction}

Platelet activation underlies thrombotic cardiovascular diseases (CVD) and events such as coronary artery disease (CAD), atherosclerosis, myocardial infarction, and thrombosis which are responsible for increased morbidity and mortality, and their prevalence in western world is increasing at a high pace [1]. Platelet activation plays a critical role in hemostasis, but inappropriate activation can lead to development of serious cardiovascular disorders, and is the causal factor in atherosclerosis [2,3]. Rupture of atherosclerotic plaque can lead to CAD development and death [4]. Therapeutic antiplatelet agents have been shown to reduce the incidence of CVD [5]. Many antiplatelet drugs, including aspirin and clopidogrel, exert side effects such as gastric bleeding and hemorrhage, limiting their usage [6]. Therefore, there is need to develop better and safe approaches to manage thrombosis and CVD. One of the approaches may include use of natural products, such as plant extracts as antithrombotic agents and anticoagulants [7]. The development of these diseases may be slowed down through the use of dietary supplements and natural products [8]. Ethnomedicinal applications are gaining popularity in the treatment of CVD [7]. Some dietary and herbal compounds have been shown to decrease the risks associated with CVD [9]. 
Morus alba L. has been traditionally used in China, Japan, Korea, and other parts of Asia as an herbal tea and medicine, and its leaves extract has been shown to exert antiplatelet effects $[10,11]$. Schisandra chinensis is known to exhibit strong anti-inflammatory and antioxidant properties. It has also long been used in traditional Chinese and Korean herbal medicine for its tonic, antioxidant, and sedative effects [1214]. In a previous report, $S$. chinensis showed antagonistic activity against platelet-activating factor (PAF) [15]. To date, there is no detailed evidence available on the antiplatelet activity of this compound. In this study, we demonstrated synergistic antithrombotic effects of these herbal compounds, which decrease platelet activity as well as thrombus formation by pharmacological suppression of platelet function.

\section{Material and Methods}

2.1. Chemicals. Collagen was purchased from Chrono-log (Havertown, PA, USA). ASA, Fura-2/AM, $\kappa$-carrageenan, and dimethyl sulfoxide (DMSO) were obtained from SigmaAldrich (St. Louis, MO, USA). ATP Assay Kit was obtained from Biomedical Research Service Center (Buffalo, NY, USA). Fibrinogen Alexa Fluor 488 conjugate was obtained from Molecular Probes (Eugene, OR, USA). Antibodies against phospho-p44/42, p44/42, phospho-p38, p38, phospho-SAPK/JNK, SAPK/JNK, phospho-Akt, and Akt were acquired from Cell Signaling Technology (Beverly, MA, USA). HPLC-grade reagents, acetonitrile, and water were obtained from J. T. Baker (Phillipsburg, NJ, USA). All chemicals were of reagent-grade.

2.2. Preparation of S. chinensis and Morus alba L. Extracts. SCC and MAL were purchased from Omniherb Co. (Daegu, Korea) and were authenticated by the classification and identification committee of Korea Institute of Oriental Medicine (KIOM) based on macroscopic and microscopic features. The same extraction procedure was applied for both plants. One kilogram of dried plant leaves was extracted twice at $80^{\circ} \mathrm{C}$ for $3 \mathrm{~h}$ with $70 \%$ ethanol (v/v). The extracts were filtered and evaporated in a rotary evaporator under low pressure. The SCC extract and MAL extract yields were $34 \%$ and $6.3 \%$, respectively. Extracts were dissolved in water and DMSO for in vivo and in vitro studies, respectively.

2.3. Animals and Dosing. Seven-week-old male Sprague Dawley rats $(n=74)$ weighing 250-260 g were purchased from Orient Co. (Seoul, Korea). Rats were acclimatized for 1 week before conducting experiments and in an air conditioned animal room with $12 \mathrm{~h}$ light and $12 \mathrm{~h}$ dark cycle at temperature and humidity of about $22 \pm 2^{\circ} \mathrm{C}$ and $55 \pm 10 \%$, respectively. Twenty-four rats were randomly divided into four groups $(n=6)$ for the arteriovenous (AV) shunt model: negative control, S. chinensis (SCC), M. alba L. (MAL), and $\mathrm{SCC}+\mathrm{MAL}$. Fifty rats were divided into five groups $(n=$ 10) for the tail thrombosis-length model: negative control, SCC, MAL, SCC + MAL, and ASA. Rats received $200 \mathrm{mg} / \mathrm{kg}$ SCC, MAL, or SCC + MAL orally once a day for 3 days
(AV shunt model groups) and 7 days (tail thrombosis-length model groups), except the negative control groups.

2.4. AV Shunt Model. The antithrombotic activity of SCC, MAL, and SCC + MAL extracts was assessed in a rat extracorporeal shunt model as previously described [16], with a little modification. Briefly, rats were administered with $200 \mathrm{mg} / \mathrm{kg}$ (human equivalence dose (HED)) [17] of SCC, MAL or SCC + MAL $(1: 1)$ extracts or vehicle orally once a day for three days. Two hours after the last administration, rats were anesthetized with urethane $(1.75 \mathrm{~g} / \mathrm{kg}$ i.p.). The left carotid artery and right jugular vein were exposed by incision and the two ends of extracorporeal shunt inserted into them. The shunt comprised of $12 \mathrm{~cm}$ polythene tubes $(0.81 \mathrm{~mm}$ external and $0.58 \mathrm{~mm}$ internal diameter $)$ joined by $5 \mathrm{~mm}$ silicone rubber plugs to a $6 \mathrm{~cm}$ polyvinyl tube with $3 \mathrm{~mm}$ of internal diameter. A cotton thread was held between two plugs so that it remained longitudinally oriented in the blood flow through cannula. The tube was filled with normal saline before cannulation and the shunt was left in place for $15 \mathrm{~min}$ after the extracorporeal circulation started. Thread was removed by stopping the blood flow to obtain thrombus that had formed, separated from thread and weighed.

2.5. $\kappa$-Carrageenan-Induced Rat Tail Thrombosis Model. To further confirm the in vivo antithrombotic activity of SCC, MAL, and SCC + MAL, a carrageenan-induced rat tail thrombosis model was used as previously described [18] with a minor modification. Rats were administered with $200 \mathrm{mg} / \mathrm{kg}$ of SCC, MAL, or SCC + MAL (1:1) extracts, $50 \mathrm{mg} / \mathrm{kg}$ of ASA, or vehicle, orally once a day for 7 days. On the 4th day, 30 min after oral administration, rats were injected with a single dose of $\kappa$-carrageenan $(20 \mathrm{mg} / \mathrm{kg} \mathrm{BW}$ i.p.) dissolved in physiological saline. Tails were observed for redness, swelling, and thrombosis. Rats were monitored for development of thrombosis and treated for a further 3 days. On the 7 th day and $72 \mathrm{~h}$ following a $\kappa$-carrageenan injection, thrombosis length was recorded for each rat in all groups.

2.6. Platelet Preparation and Aggregation. Whole blood was obtained from SD rats and transferred to anticoagulant acid/citrate/dextrose (ACD) solution ( $21 \mathrm{mM}$ citric acid, $85 \mathrm{mM}$ trisodium citrate, and $83 \mathrm{mM}$ dextrose) containing tubes. Platelet rich plasma (PRP) was obtained by centrifuging the blood at $170 \times \mathrm{g}$ for $7 \mathrm{~min}$ and PRP was again centrifuged two times at $350 \times \mathrm{g}$ to isolate washed platelets. Tyrode's buffer solution ( $137 \mathrm{mM} \mathrm{NaCl}, 12 \mathrm{mM} \mathrm{NaHCO}$, $5.5 \mathrm{mM}$ glucose, $2 \mathrm{mM} \mathrm{KCl,} 1 \mathrm{mM} \mathrm{MgCl}$, and $\mathrm{NaHPO}_{4}$, $\mathrm{pH}$ 7.4) was added to adjust the platelet concentration at $3 \times$ $10^{8} / \mathrm{mL}$ to proceed for platelet aggregation assay. All the procedure for preparation was conducted at room temperature $\left(23 \pm 2^{\circ} \mathrm{C}\right)$.

Platelet aggregation was performed as previously described method [19] and the extent of aggregation was measured by light transmission aggregometry (Chrono-log, Havertown, PA, USA). Washed platelets were preincubated 
for $2 \mathrm{~min}$ at $37^{\circ} \mathrm{C}$ along with $1 \mathrm{mM} \mathrm{CaCl} 2$ and stimulated with collagen; then solution was incubated for more $5 \mathrm{~min}$ with continuous stirring. The vehicle ratio was not more than $0.05 \%$.

2.7. Scanning Electron Microscopy Analysis. Field Emission Scanning Electron Microscope (SU8220, Hitachi) was used to assess aggregation ultrastructure image at center for scientific instrument, Kyungpook National University, Daegu, Korea. After the platelet aggregation assay, the platelets mixtures were fixed using $0.5 \%$ paraformaldehyde (1st fixation) and osmium tetroxide (2nd fixation), dehydrated using ethanol, freeze-dried, and scanned.

2.8. $\left[\mathrm{Ca}^{2+}\right]_{i}$ Measurement. $\left[\mathrm{Ca}^{2+}\right]_{i}$ concentration was measured with Fura-2/AM as previously described [20]. Briefly, platelets were preincubated with $5 \mu \mathrm{M}$ Fura-2/AM for $1 \mathrm{~h}$ at $37^{\circ} \mathrm{C}$ and washed. Platelets were then preincubated for $1 \mathrm{~min}$ along with $1 \mathrm{mM} \mathrm{CaCl}{ }_{2}$ at $37^{\circ} \mathrm{C}$ and stimulated with agonist for $2 \mathrm{~min}$. Fluorescence was recorded using spectrofluorometer (F-2500; Hitachi, Japan) and $\left[\mathrm{Ca}^{2+}\right]_{i}$ was calculated as previously described method by Schaeffer and Blaustein [21] using the following formula: $\left[\mathrm{Ca}^{2+}\right]_{i}$ in cytosol $=224 \mathrm{nM} \times(F-$ $\left.F_{\min }\right) /\left(F_{\max }-F\right)$, in which $224 \mathrm{nM}$ is the dissociation constant of the Fura-2-Ca ${ }^{2+}$ complex and $F_{\min }$ and $F_{\max }$ represent the fluorescence intensity levels at very low and very high $\mathrm{Ca}^{2+}$ concentrations, respectively.

2.9. Granule Secretion. Washed platelets were preincubated along with $1 \mathrm{mM} \mathrm{CaCl}$ for $2 \mathrm{~min}$ at $37^{\circ} \mathrm{C}$ and stimulated with agonist. Supernatants were obtained by centrifuging the platelet mixture after the aggregation reaction terminated, and ATP secretion was measured in a luminometer (GloMax 20/20, Promega, Madison, WI, USA) using an ATP Assay Kit (Biomedical Research Service Center).

2.10. Flow Cytometry. Flow cytometry was used to quantify fibrinogen Alexa Fluor 488 conjugate binding to platelets. Washed platelets were preincubated for $2 \mathrm{~min}$ along with $0.1 \mathrm{mM} \mathrm{CaCl}_{2}$, stimulated with agonist for $5 \mathrm{~min}$ and then incubated with fibrinogen Alexa Fluor $488(20 \mu \mathrm{g} / \mathrm{ml})$ for $5 \mathrm{~min}$ at room temperature, and then fixed with $0.5 \%$ paraformaldehyde for $30 \mathrm{~min}$ at $4^{\circ} \mathrm{C}$. Platelets were suspended in $400 \mu \mathrm{L}$ phosphate buffered saline (PBS) after three times washing with PBS. The fluorescence was analyzed using a FACS Caliber cytometer (BD Biosciences, San Jose, CA, USA), and data were analyzed using CellQuest software (Becton Dickinson Immunocytometry Systems, San Jose, CA, USA).

2.11. Immunoblotting. After platelet aggregation, suspension was solubilized in sample lysis buffer $(0.125 \mathrm{M}$ Tris- $\mathrm{HCl}$ [pH 6.8], 2\% SDS, 2\% $\beta$-mercaptoethanol, $20 \%$ glycerol, $0.02 \%$ bromophenol blue, $1 \mu \mathrm{g} / \mathrm{mL}$ phenyl methyl sulfonyl fluoride [PMSF], $2 \mu \mathrm{g} / \mathrm{mL}$ aprotinin, $1 \mu \mathrm{g} / \mathrm{mL}$ leupeptin, and $1 \mu \mathrm{g} / \mathrm{mL}$ pepstatin A) and centrifuged. Protein concentrations were measured using BCA assay (PRO-MEASURE; iNtRON Biotechnology, Seoul, Korea) and total cell proteins
$(35 \mu \mathrm{g})$ were isolated from lysates using 10\% SDS-PAGE and transferred to polyvinylidene difluoride membranes in transfer buffer $(25 \mathrm{mM}$ Tris [pH 8.3], $0.2 \mathrm{M}$ glycine, and $20 \%$ methanol). Membranes were blocked in TBS-T containing 5\% dry skim milk and probed with primary and secondary antibodies, and then antibody binding was visualized using enhanced chemiluminescence (Advansta, CA, USA).

2.12. High-Performance Liquid Chromatography (HPLC) Analysis to Identify the Marker Compounds in SCC + MAL. The mixture was analyzed by reverse phase-HPLC (Waters Alliance 2695 system, Waters Co., Milford, MA, USA) combined with a 2996-photodiode array detector. A Phenomenex Luna C18 column $(250 \times 4.6 \mathrm{~mm} ; 5 \mu \mathrm{m}$ particle size, Phenomenex, Torrance, CA, USA) was used for the stationary phase; the mobile phase was composed of $0.1 \%(\mathrm{v} / \mathrm{v})$ trifluoroacetic aqueous solution (A) and acetonitrile (B). The elution conditions were as follows: at $t=0 \mathrm{~min}$, the mobile phase was held for $10 \mathrm{~min}$ and consisted of $90 \% \mathrm{~A}$ and $10 \% \mathrm{~B}$. A gradient was applied for 10 to $60 \mathrm{~min}$, to $40 \% \mathrm{~A}$ and $60 \% \mathrm{~B}$, which was followed by a wash with $100 \%$ B for 10 min and a 15 min equilibration period at $90 \% \mathrm{~A}$ and $10 \% \mathrm{~B}$. A flow rate of $1.0 \mathrm{~mL} / \mathrm{min}$ at a temperature of $40^{\circ} \mathrm{C}$, and an injection volume of $20 \mu \mathrm{L}$ were maintained throughout the analysis for the separation.

The compounds were recognized by comparing retention times and UV spectra of the peaks of HPLC/PDA chromatograms to those of commercially available standards. For each compound, peak area was determined at a wavelength of $240 \mathrm{~nm}$. The calibration curve of the standards, ranging from 12.5 to $200 \mu \mathrm{g} / \mathrm{mL}$ (5 levels) for each standard, revealed a good linearity with the linear correlation coefficient; $R^{2}$ values exceeded 0.99 (peak area versus concentration). Quantitation was performed in comparison to a mixture of external standards of known concentrations that was analyzed in duplicate before and after the batch samples and the peak areas were used to calculate the concentrations of the compounds in the samples.

2.13. Statistical Analysis. One-way analysis of variance (ANOVA) followed by post hoc Dunnett's test was used to determine statistical significance in the observed differences (SAS Institute Inc., Cary, NC, USA). All data are presented as the mean \pm standard error of the mean (SEM) and $P$ value of 0.05 or less was considered statistically significant.

\section{Results}

3.1. In Vivo Effects on Thrombus Formation. AV shunt thrombosis models have been used to evaluate in vivo antithrombotic effects $[3,16]$. We therefore studied the effects of SCC, MAL, and SCC + MAL on thrombus formation in an extracorporeal shunt model. As shown in Figure 1(a), all treated groups displayed significantly reduced arteriovenous shunt thrombus formation compared to that observed for negative controls $(P<0.001)$. Moreover, the mixture showed more potent thrombus reduction $(47 \pm 2.3 \%)$ than SCC or 


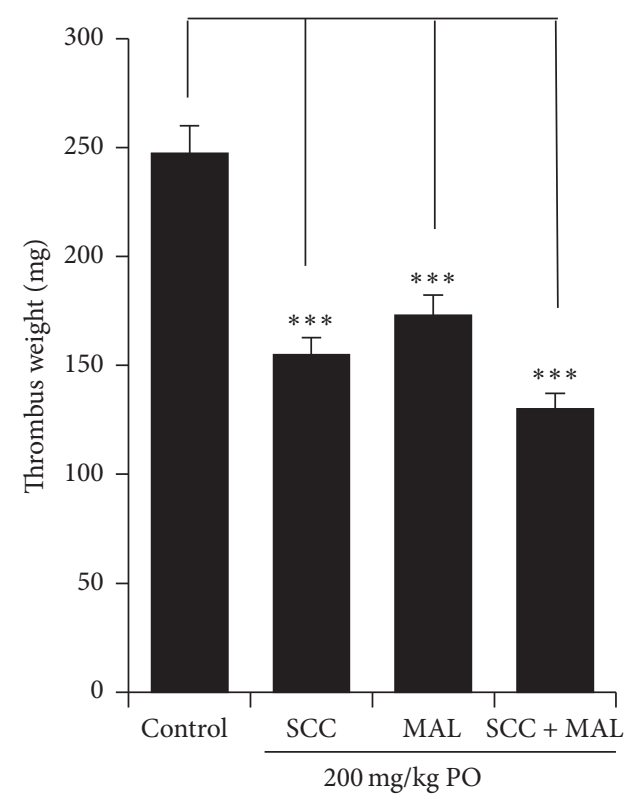

(a)

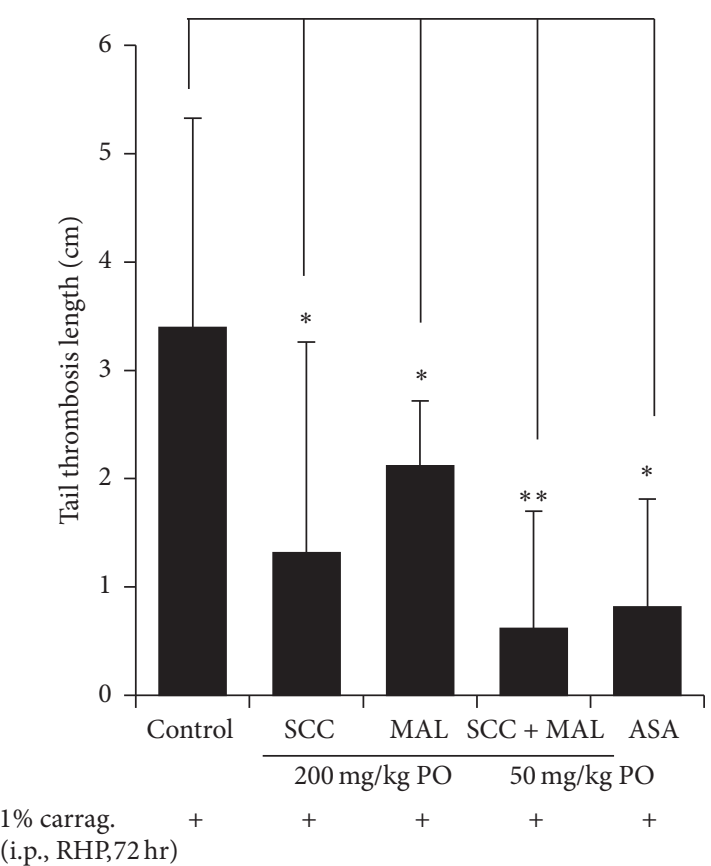

(b)

FIGURE 1: In vivo effects of the SCC + MAL mixture on thrombus formation. (a) The arteriovenous (AV) shunt model was used to evaluate in vivo antithrombotic activity. Rats received SCC, MAL, or SCC + MAL extracts or vehicle, orally once a day for 3 days. On the $3 \mathrm{rd}$ day, $2 \mathrm{~h}$ after oral administration, blood circulation in the cannula carried out for $15 \mathrm{~min}$, and thrombus weight determined immediately. (b) Rat tail thrombosis-length model was used to evaluate inhibitory effects on tail thrombosis. Rats received SCC, MAL, or SCC + MAL extracts, ASA, or vehicle, orally once a day for 7 days. On the 4 th day, $30 \mathrm{~min}$ after oral administration, rats were injected with a single dose of $\kappa$-carrageenan $(20 \mathrm{mg} / \mathrm{kg}$ BW i.p.) dissolved in physiological saline. Bar graph shows the mean \pm SEM for AV shunt $(n=3$ rats in each group) and tail thrombosis $\left(n=10\right.$ rats in each group). ${ }^{*} P<0.05,{ }^{* *} P<0.01$, and ${ }^{* * *} P<0.001$ versus control.

MAL $(37 \pm 2.3$ or $32 \pm 0.2 \%$, respectively) alone $\operatorname{did}(P<$ 0.001).

A $\kappa$-carrageenan-induced tail thrombosis-length model in rats is useful for evaluating antithrombotic effects [22]. We measured and recorded thrombus length; swelling and redness were observed in the negative control groups within 4-5 h of $\kappa$-carrageenan injection, but not in the treatment groups. Length of tail thrombosis was recorded $72 \mathrm{~h}$ after $\kappa$ carrageenan injection. As shown in Figure 1(b), the negative control group displayed a significant increase in thrombosis length compared to that observed in rats treated with ASA $(77 \pm 21 \% ; P<0.05)$, which was used as a reference antithrombotic agent. The SCC and MAL groups showed notable decreases in thrombosis length, that is, $62 \pm 39 \%$ and $39 \pm$ $11 \%(P<0.05)$, respectively, compared with control, while the SCC + MAL group displayed a significant reduction in thrombosis length $(83 \pm 22 \% ; P<0.01)$ compared to that reported for all other groups, indicating that the mixture exerted synergistic inhibitory effects on $\kappa$-carrageenaninduced thrombus formation and had potent antithrombotic properties.

3.2. Effects on Agonist-Induced Platelet Aggregation. To further confirm the antiplatelet activity of SCC + MAL, we investigated the underlying mechanism using in vitro techniques. Light transmission aggregometry was employed to assess the effect of SCC + MAL on agonist-induced platelet aggregation. First of all we evaluated whether our compound affected various ligands (ADP, collagen, or thrombin) but SCC + MAL only inhibited collagen-induced platelet aggregation (data not shown). It is also consistent with our previous study [11], which have shown that MAL only inhibited collageninduced platelet aggregation and did not affect other ligands. Moreover, we tried different dosage of SCC + MAL and $400 \mu \mathrm{g} / \mathrm{ml}$ found to inhibit maximum platelet aggregation (data not shown). Therefore, we employed collagen as ligand and found that SCC + MAL dramatically inhibited collageninduced platelet aggregation by $94 \pm 03 \%$ compared to the control value $(P<0.001)$ (Figure 2$)$.

3.3. Effects on $\left[\mathrm{Ca}^{2+}\right]_{i}$. $\left[\mathrm{Ca}^{2+}\right]_{i}$ elevation plays a vital role in platelet aggregation and degranulation and modulates platelet functions such as thrombus formation and platelet shape change [14]. To assess whether the SCC + MAL mixture inhibited $\left[\mathrm{Ca}^{2+}\right]_{i}$, an intracellular calcium mobilization assay was performed. As shown in Figure 3, SCC + MAL completely blocked $\left[\mathrm{Ca}^{2+}\right]_{i}$ mobilization $(P<0.001)$.

3.4. Effects on Granule Secretion (ATP Release). Platelet activation causes the release of different granule populations such as $\alpha$-granules and dense granules; these secretions boost 


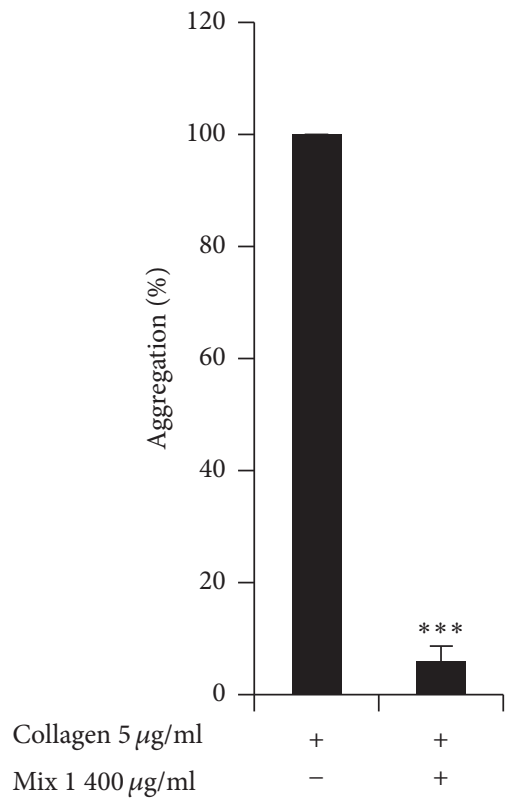

(a)

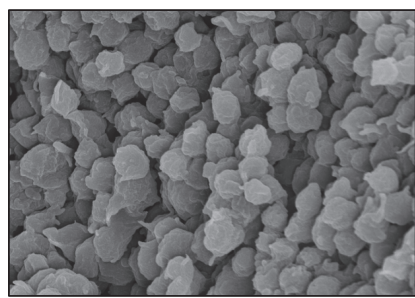

(A)

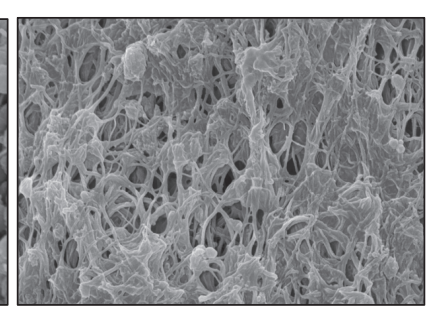

(B)

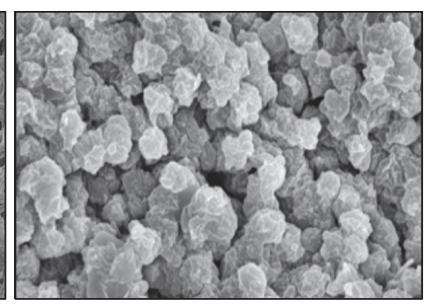

(C)

(b)

Figure 2: (a) SCC + MAL inhibited collagen-induced platelet aggregation. Aggregation was quantified and expressed as a percentage. Graph shows the mean \pm SEM of at least four independent experiments. (b) Representative scanning electron microscopy images of resting state or collagen-treated platelets incubated with or without SCC + MAL: basal (A), $5 \mu \mathrm{g} / \mathrm{mL}$ of collagen (B), and $400 \mu \mathrm{g} / \mathrm{mL}$ of SCC + MAL (C). ${ }^{* * *} P<0.001$ compared to the agonist control.

platelet activation and intracellular signaling. We examined the effects of SCC + MAL on ATP release from dense granules and found that the mixture inhibited ATP release by $47 \pm 15 \%$ $(P<0.001)$ (Figure 4$)$.

3.5. SCC + MAL Limits Integrin $\alpha_{I I b} \beta_{3}$ Mediated Inside-Out Signaling. Platelets express integrins, such as $\alpha_{2} \beta_{1}$ (collagen receptor), $\alpha_{\mathrm{IIb}} \beta_{3}$ (fibrinogen receptor), and $\alpha_{\mathrm{V}} \beta_{1}$ (fibronectin receptor). The fibrinogen binding to integrin $\alpha_{\mathrm{IIb}} \beta_{3}$ is considered a marker of $\alpha_{\text {IIb }} \beta_{3}$ inside-out signaling [23]. We investigated the effect of SCC + MAL on fibrinogen binding to integrin $\alpha_{\mathrm{II}} \beta_{3}$ and found that SCC + MAL notably reduced fibrinogen binding to integrin $\alpha_{\text {IIb }} \beta_{3}$ by $65 \pm 06 \%(P<0.001)$ (Figure 5).

3.6. SCC + MAL Reduced MAPK and Akt Phosphorylation. To explore the underlying intracellular signaling pathway of SCC + MAL effects on platelet activation, we evaluated its effect on protein phosphorylation. Phosphorylation of MAPKs (JNK, ERK, and P38-MAPK) is known to mediate the platelet activation pathway [24] while PI3K/Akt signaling is another important pathway in platelet activation. Therefore we investigated whether SCC + MAL decreased MAPK and PI3K/Akt phosphorylation. SCC + MAL markedly blocked collagen-induced phosphorylation of MAPKs (ERK and JNK but not P38-MAPK) and Akt, indicating that the mixture can affect the MAPK and PI3K/Akt signaling pathways (Figure 6).

3.7. Chromatographic Separation of SCC + MAL to Identify Marker Compounds. Marker compounds were determined from the major peaks of HPLC/PDA chromatograms in comparison with the retention times and UV spectra of commercial standards. HPLC analysis of SCC + MAL identified four compounds: isoquercitrin and astragalin were derived from M. alba L., and schizandrol A and gomisin A were derived from $S$. chinensis; these appeared at a retention time of approximately $25.6,28.0,51.6$, and $55.1 \mathrm{~min}$, respectively (Figure 7). SCC + MAL contained $13.6 \mathrm{mg} / \mathrm{g}$ of isoquercitrin, $5.8 \mathrm{mg} / \mathrm{g}$ of astragalin, $7.3 \mathrm{mg} / \mathrm{g}$ of schizandrol A, and $2.0 \mathrm{mg} / \mathrm{g}$ of gomisin A. 


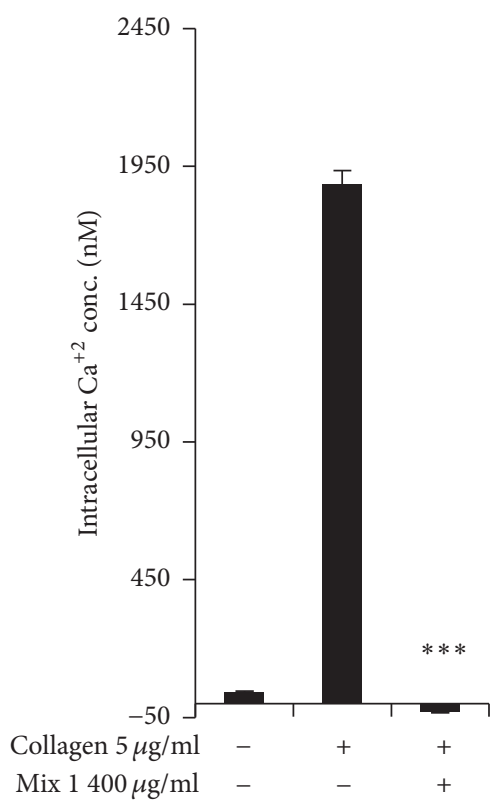

FIGURE 3: The inhibitory effect of SCC + MAL on $[\mathrm{Ca} 2+]_{i}$ induced by collagen. Washed platelets $\left(3 \times 10^{8} / \mathrm{mL}\right)$ were incubated with a calcium fluorophore $(5 \mu \mathrm{M}$, Fura-2/AM) and stimulated with collagen $(5 \mu \mathrm{g} / \mathrm{mL})$. The results are presented as the mean \pm SEM of at least four independent experiments. ${ }^{* * *} P<0.001$ compared to the agonist control.

\section{Discussion}

Coronary atherosclerotic disease and other acute clinical manifestations are commonly caused by plaque disruption and subsequent thrombus formation [4]. Previous studies have suggested that prophylactic pharmacological suppression of platelet activation can inhibit the risk of prothrombotic state, slow down the development of thrombosis, and reduce the risk of CVD $[5,25]$. Findings from primary prevention trials have noted that the side effects of some prophylactic regimens outweigh their benefits [26, 27]. Several studies reported that certain dietary and herbal compounds can reduce the risk factors linked to CVD [7, 9].

MAL has traditionally been used as an herbal medicine and has recently been shown to have antiplatelet and antithrombotic properties [11]. SCC has been used as a Korean herbal medicine for its antitussive, antioxidant, sedative, and tonic effects. SCC fruits and leaves contain a number of pharmacologically active compounds [28]. We therefore evaluated its antiplatelet and antithrombotic properties in combination with MAL and explored the underlying mechanism of their effects on platelet function modulation, by assessing the in vivo antithrombotic activity of both extracts individually and as a mixture.

Carrageenan, which is composed of repeating units of 3,6-anhydro-D-galactose and $\mathrm{D}$-galactose, is a straightchain, sulfur-containing macromolecular polysaccharide. $\kappa$ carrageenan is broadly used for induction of tissue inflammation and tail thrombosis in laboratory animals such as rats and mice. It can cause endothelial cell injury and local

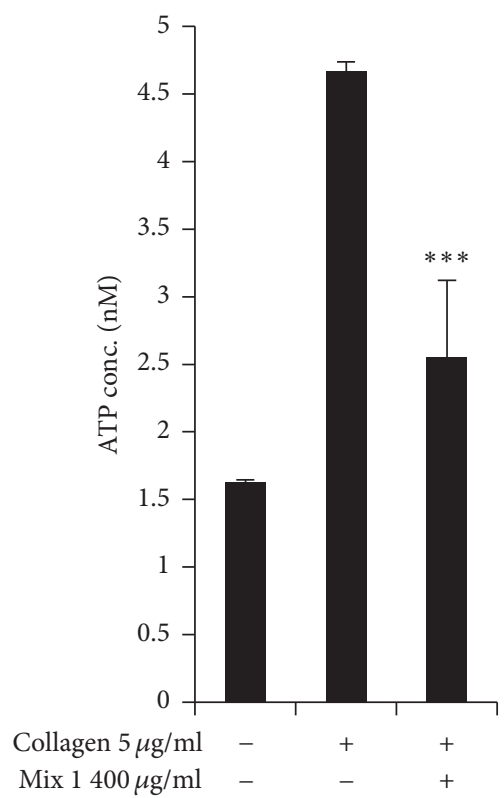

FIGURE 4: Inhibitory effects of SCC + MAL on dense granules secretions. Washed platelets $\left(3 \times 10^{8} / \mathrm{mL}\right)$ were preincubated for $2 \mathrm{~min}$ at $37^{\circ} \mathrm{C}$ in the presence of $1 \mathrm{mM} \mathrm{CaCl}_{2}$ and then stimulated with collagen. After terminating the aggregation, ATP release assay was performed. Bar graph shows the mean \pm SEM of at least four independent experiments. ${ }^{* * *} P<0.001$ compared to the agonist control.

blood vessel inflammation by releasing inflammatory mediators, which may lead to thrombus formation [29]. Blood vessel inflammation is considered one cause of thrombosis, although a thrombus can lead to inflammation [4]. Circulation disturbances at locations of atherosclerotic plaque rupture stimulate platelet activation and arterial thrombus formation [30]. In this study, we evaluated both extracts in vivo against thrombus formation and found SCC + MAL to be a potent combination compared with SCC or MAL alone; the mixture may be a natural antithrombotic agent with potential to prevent thrombosis or CVD.

SCC + MAL markedly inhibited collagen-induced platelet aggregation through glycoprotein (GP) VI-mediated signaling in platelets. A previous report described MAL $(400 \mu \mathrm{g} / \mathrm{mL})$ inhibition of collagen $(2.5 \mu \mathrm{g} / \mathrm{mL})$-induced platelet aggregation by 30\% [11]. Our results show more potent inhibition of platelet aggregation with SCC + MAL $(94 \pm 3 \%)$ at similar concentrations as $5 \mu \mathrm{g} / \mathrm{mL}$ collagen, indicating that SCC + MAL is an effective natural antiplatelet agent. Cytosolic calcium elevation is mediated through cytosolic release and influx across the plasma membrane [31]. $\left[\mathrm{Ca}^{2+}\right]_{i}$ plays a crucial role in platelet activation and increased calcium levels lead to the activation of numerous signaling pathways involved in actin-myosin interaction [32]. We found that SCC + MAL significantly inhibited $\left[\mathrm{Ca}^{2+}\right]_{i}$ concentration in collagen-induced platelet aggregation.

Our study demonstrates the inhibitory potential of SCC + MAL on collagen-stimulated platelet aggregation, which is indicated by a clear suppression of $\left[\mathrm{Ca}^{2+}\right]_{i}$ mobilization, 


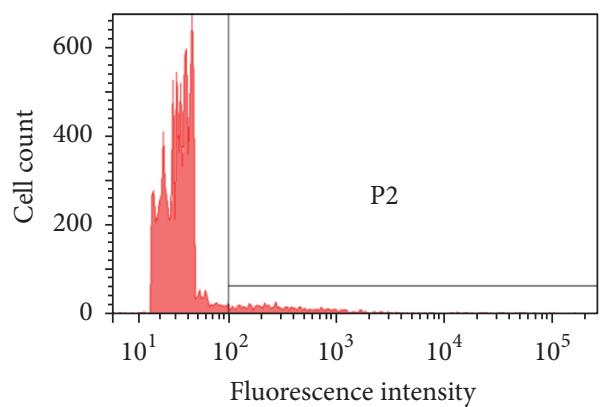

(A)

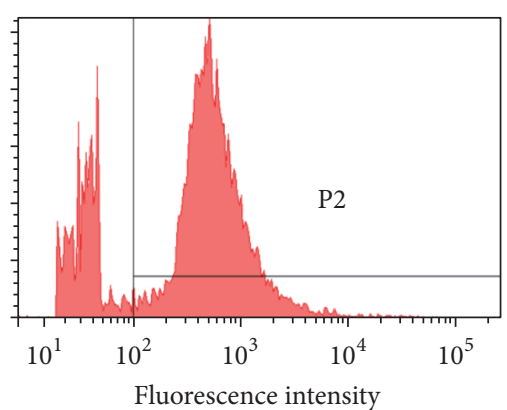

(B)

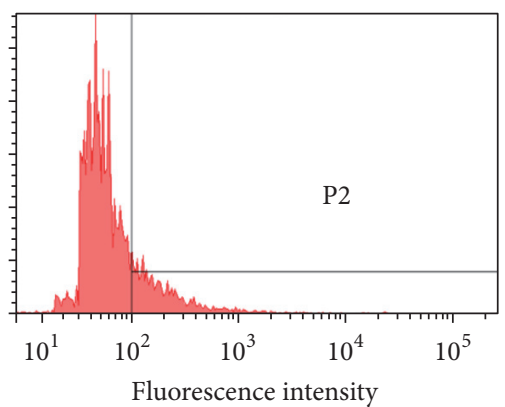

(C)

(a)

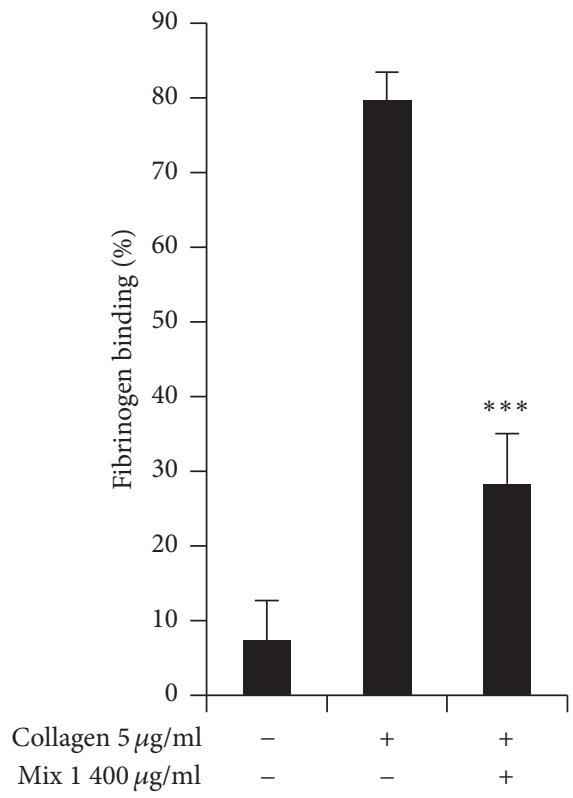

(b)

FIGURE 5: (a) The inhibitory effect of SCC + MAL on fibrinogen binding to integrin $\alpha_{\text {IIb }} \beta_{3}$. Washed platelets $\left(3 \times 10^{8} / \mathrm{mL}\right)$ were preincubated for $2 \mathrm{~min}$ at room temperature in the presence of $0.1 \mathrm{mM} \mathrm{CaCl}_{2}$, stimulated with collagen for $5 \mathrm{~min}$ and fibrinogen Alexa Fluor $488(20 \mu \mathrm{g} / \mathrm{mL})$, and then fixed with $0.5 \%$ paraformaldehyde at $4{ }^{\circ} \mathrm{C}$ for $30 \mathrm{~min}$. Representative FACS analysis results of four independent trials are shown. Basal (A), $5 \mu \mathrm{g} / \mathrm{mL}$ of collagen (B), and $400 \mu \mathrm{g} / \mathrm{mL}$ of SCC + MAL (C). (b) Bar graph summarizing the inhibitory effect of SCC + MAL extract on fibrinogen binding. ${ }^{* * *} P<0.001$ compared to the agonist control.

granule secretion, and integrin $\alpha_{\mathrm{IIb}} \beta_{3}$ activation. Fibrinogen binding to integrin $\alpha_{\mathrm{IIb}} \beta_{3}$ is considered a marker of inside-out signaling; we measured the binding using flow cytometry in the presence or absence of SCC + MAL. Our findings suggest that compromised $\alpha_{\mathrm{IIb}} \beta_{3}$ structural changes may be induced by pretreatment with SCC + MAL. Conversely, these results also imply that outside-in signaling, leading to platelet shape change, may be reduced by SCC + MAL treatment. Platelets are continually exposed to a number of activating factors, such as collagen, thrombin, ADP, fibrinogen, vWF, and inhibitory factors such as prostacyclin $\left(\mathrm{PGI}_{2}\right)$ and ADPase [33]. Thrombosis can be developed if there is disturbance in this equilibrium. Accordingly, for a normal hemostasis, a resilient equilibrium between two routes of platelet activation and inhibition should be critical. Our study proposes that pretreatment of stimulated platelets with SCC + MAL may assist in maintaining this equilibrium.

We observed that SCC + MAL inhibited collagenstimulated ERK1/2 and JNK but not p38-MAPK activation. A strong inhibitory effect on PI3K/Akt signaling was also observed, showing that both pathways may be involved in modulation of SCC + MAL antiplatelet activity. PI3K inhibitors have been shown to prevent fibrinogen binding to integrin $\alpha_{\mathrm{IIb}} \beta_{3}$ and thrombus formation without affecting coagulation parameters [34]. Previous findings established that PI3K/Akt pathway plays a key role in cardiac protection by inducing antiapoptotic effects and reducing myocardial ischemia-reperfusion injury (MI/RI) $[35,36]$. SCC + MAL may potentially exhibit beneficial effects by modulation of PI3K/Akt signaling in reperfusion injury salvage kinase 


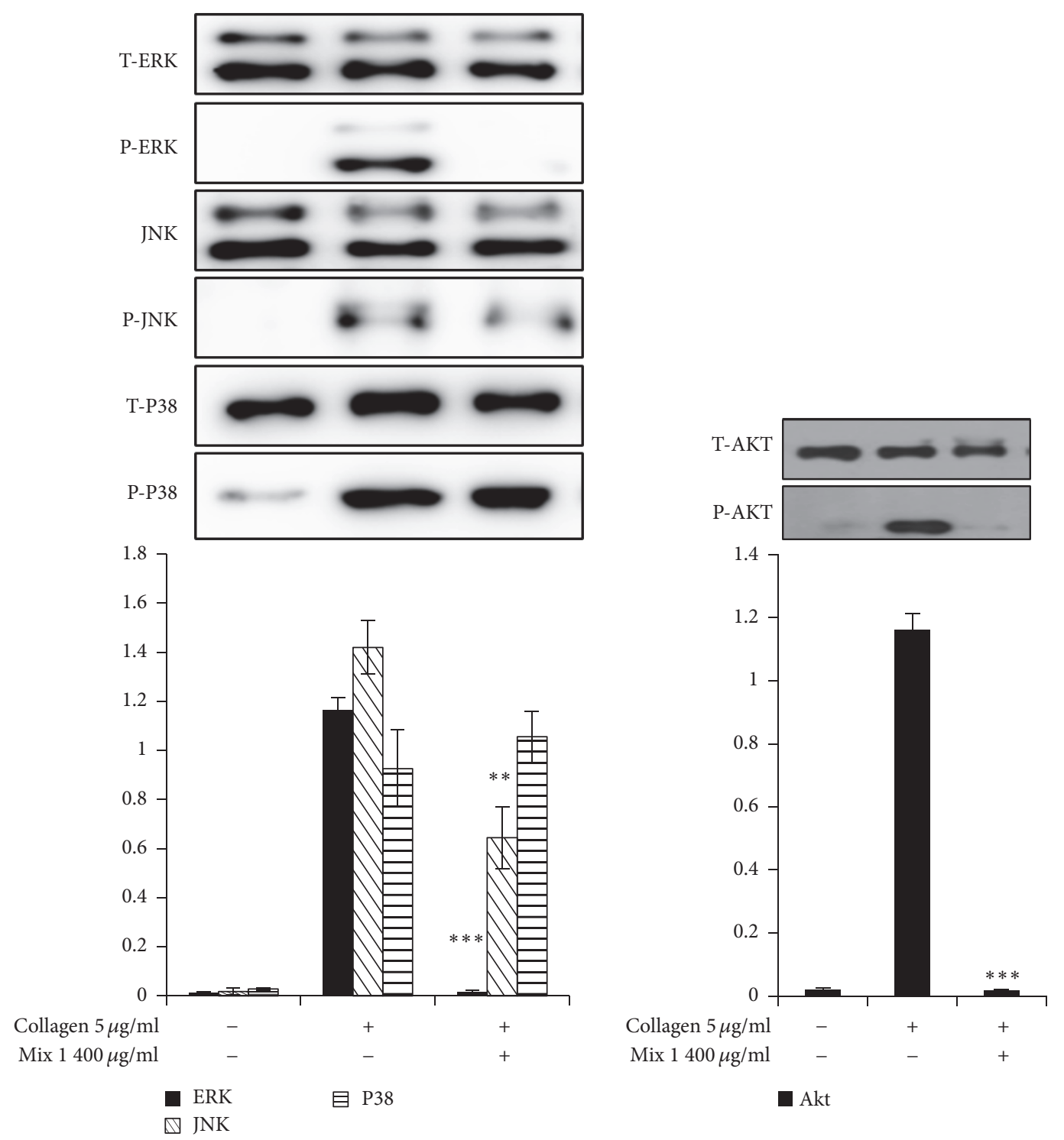

FIGURE 6: SCC + MAL reduced the phosphorylation of MAPK (i.e., ERK1/2 and JNK but not p38) and Akt. Cell proteins were extracted after aggregation was terminated, and proteins were separated using SDS-PAGE, transferred to PVDF membranes, and then probed with antibodies against total and phospho-ERK1/2, JNK, p38, and Akt. All immunoblots were carried out in at least four independent experiments. ${ }^{* *} P<0.01$ and ${ }^{* * *} P<0.001$ compared to the agonist-treated group.

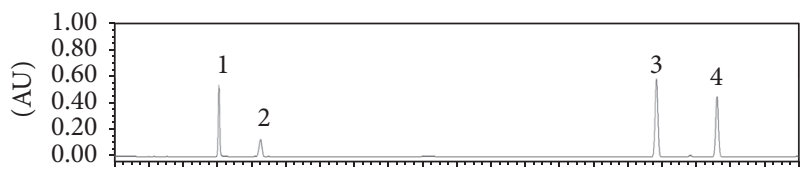

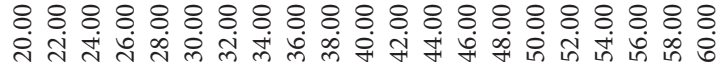
Minutes

(a)

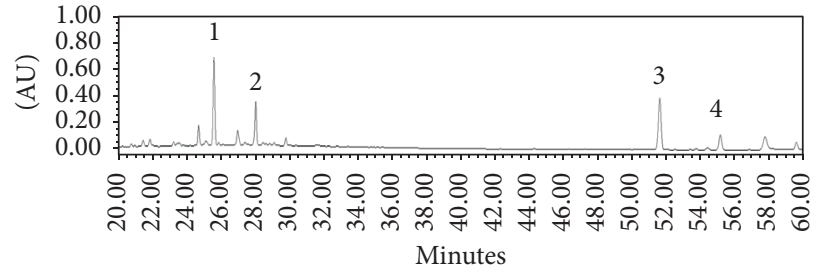

(b)

FIGURE 7: HPLC chromatograms of four standards mixture (a) at $240 \mathrm{~nm}$ and the $70 \%$ (v/v) ethanol extract of SCC + MAL. Isoquercitrin (1), astragalin (2), schizandrol A (3), and gomisin A (4) appeared at a retention time of approximately 25.6, 28.0, 51.6, and 55.1 min, respectively. 
(RISK) pathway. Platelets present MAPK pathway factors such as JNK, ERK and P38 which can be activated by different agonists [24]. Collagen-stimulated ERK2 activation is reliant on $\mathrm{TXA}_{2}$ production and ADP secretion [37].

Our findings suggest that SCC + MAL can suppress the GP VI downstream signaling pathway; hence, antagonism of this receptor may characterize a novel therapeutic regimen. Chromatographic separation led to the identification of four compounds in SCC + MAL mixture. Schizandrol A from SCC may be the one that synergistically enhances the antiplatelet effects of MAL. The SCC + MAL mixture inhibited thrombus formation, agonist-stimulated aggregation, $\left[\mathrm{Ca}^{2+}\right]_{i}$ mobilization, granule secretion, and $\alpha_{\mathrm{II}} \beta_{3}$ activation via MAPK and Akt phosphorylation, demonstrating a potential use of this mixture as an ethnomedicinal antithrombotic agent.

\section{Conclusion}

The SCC + MAL mixture significantly inhibited in vivo thrombus formation and was a potent inhibitor of collageninduced in vitro platelet aggregation and granule secretion. Our findings indicate that SCC + MAL mixture inhibits agonist-stimulated platelet activation through modulation of downstream signaling via the MAPK and PI3K/Akt pathways.

\section{Disclosure}

Dong-Seon Kim and Muhammad Irfan are co-first authors.

\section{Competing Interests}

The authors have no conflict of interests.

\section{Authors' Contributions}

Dong-Seon Kim and Muhammad Irfan equally contributed to this work.

\section{Acknowledgments}

This work was supported by the National Research Foundation of Korea grant funded by the Korean government (Grant no. 2015R1D1A1A09057204) and by the High ValueAdded Food Technology Development Program (314043-3), Ministry of Agriculture, Food and Rural Affairs, Republic of Korea.

\section{References}

[1] D. Mozaffarian, E. J. Benjamin, A. S. Go et al., "Heart disease and stroke statistics-2016 update: a report from the American Heart Association," Circulation, vol. 133, no. 4, pp. e38-360, 2016.

[2] G. Vilahur and L. Badimon, "Antiplatelet properties of natural products," Vascular Pharmacology, vol. 59, no. 3-4, pp. 67-75, 2013.

[3] M. Endale, W. M. Lee, S. M. Kamruzzaman et al., "GinsenosideRp1 inhibits platelet activation and thrombus formation via impaired glycoprotein VI signalling pathway, tyrosine phosphorylation and MAPK activation," British Journal of Pharmacology, vol. 167, no. 1, pp. 109-127, 2012.

[4] M. Sadowski, M. Zabczyk, and A. Undas, "Coronary thrombus composition: links with inflammation, platelet and endothelial markers," Atherosclerosis, vol. 237, no. 2, pp. 555-561, 2014.

[5] Antithrombotic Trialists' Collaboration, "Collaborative metaanalysis of randomised trials of antiplatelet therapy for prevention of death, myocardial infarction, and stroke in high risk patients," British Medical Journal, vol. 324, no. 7329, pp. 71-86, 2002.

[6] D. L. Bhatt and E. J. Topol, "Scientific and therapeutic advances in antiplatelet therapy," Nature Reviews Drug Discovery, vol. 2, no. 1, pp. 15-28, 2003.

[7] S. Rastogi, M. M. Pandey, and A. K. S. Rawat, "Traditional herbs: a remedy for cardiovascular disorders," Phytomedicine, vol. 23, no. 11, pp. 1082-1089, 2016.

[8] F. B. Hu, E. B. Rimm, M. J. Stampfer, A. Ascherio, D. Spiegelman, and W. C. Willett, "Prospective study of major dietary patterns and risk of coronary heart disease in men," The American Journal of Clinical Nutrition, vol. 72, no. 4, pp. 912-921, 2000.

[9] A. K. Dutta-Roy, "Dietary components and human platelet activity," Platelets, vol. 13, no. 2, pp. 67-75, 2002.

[10] F. B. Hu, "Plant-based foods and prevention of cardiovascular disease: an overview," The American Journal of Clinical Nutrition, vol. 78, supplement 3, pp. 544S-551S, 2003.

[11] D.-S. Kim, H. D. Ji, M. H. Rhee et al., "Antiplatelet activity of morus alba leaves extract, mediated via inhibiting granule secretion and blocking the phosphorylation of extracellularsignal-regulated kinase and akt," Evidence-based Complementary and Alternative Medicine, vol. 2014, Article ID 639548, 11 pages, 2014.

[12] D.-F. Chen, S.-X. Zhang, L. Xie et al., "Anti-AIDS agentsXXVI. Structure-activity correlations of gomisin-G-related anti-HIV lignans from Kadsura interior and of related synthetic analogues," Bioorganic and Medicinal Chemistry, vol. 5, no. 8, pp. 1715-1723, 1997.

[13] P. Y. Chiu, D. H. F. Mak, M. K. T. Poon, K. M. Ko, and R. Ko, "In vivo antioxidant action of a lignan-enriched extract of Schisandra fruit and an anthraquinone-containing extract of Polygonum root in comparison with schisandrin B and emodin," Planta Medica, vol. 68, no. 11, pp. 951-956, 2002.

[14] P. Y. Chiu and K. M. Ko, "Schisandrin B protects myocardial ischemia-reperfusion injury partly by inducing Hsp25 and Hsp70 expression in rats," Molecular and Cellular Biochemistry, vol. 266, no. 1-2, pp. 139-144, 2004.

[15] K. Y. Jung, I. S. Lee, S. R. Oh, D. S. Kim, and H.-K. Lee, "Lignans with platelet activating factor antagonist activity from Schisandra chinensis (Turcz.) Baill," Phytomedicine, vol. 4, no. 3, pp. 229-231, 1997.

[16] T. Umetsu and K. Sanai, "Effect of 1-methyl-2-mercapto-5-(3pyridyl)-imidazole (KC-6141), an anti-aggregating compound, on experimental thrombosis in rats," Thrombosis and Haemostasis, vol. 39, no. 1, pp. 74-83, 1978.

[17] J.-W. Shin, I.-C. Seol, and C.-G. Son, "Interpretation of animal dose and human equivalent dose for drug development," The Journal of Korean Medicine, vol. 31, pp. 1-7, 2010.

[18] H. Bekemeier and A. J. Giessler, "Thrombosis induction by different carrageenans in rats and mice," Naturwissenschaften, vol. 74, no. 7, pp. 345-346, 1987. 
[19] H.-S. Lee, S. D. Kim, W. M. Lee et al., "A noble function of BAY 11-7082: inhibition of platelet aggregation mediated by an elevated cAMP-induced VASP, and decreased ERK2/JNK1 phosphorylations," European Journal of Pharmacology, vol. 627, no. 1-3, pp. 85-91, 2010.

[20] S. M. Kamruzzaman, M. Endale, W. J. Oh et al., "Inhibitory effects of Bulnesia sarmienti aqueous extract on agonist-induced platelet activation and thrombus formation involves mitogenactivated protein kinases," Journal of Ethnopharmacology, vol. 130, no. 3, pp. 614-620, 2010.

[21] J. Schaeffer and M. P. Blaustein, "Platelet free calcium concentrations measured with fura- 2 are influenced by the transmembrane sodium gradient," Cell Calcium, vol. 10, no. 2, pp. 101-113, 1989.

[22] R. Arslan, Z. Bor, N. Bektas, A. H. Meriçli, and Y. Ozturk, "Antithrombotic effects of ethanol extract of Crataegus orientalis in the carrageenan-induced mice tail thrombosis model," Thrombosis Research, vol. 127, no. 3, pp. 210-213, 2011.

[23] S. J. Shattil and P. J. Newman, "Integrins: dynamic scaffolds for adhesion and signaling in platelets," Blood, vol. 104, no. 6, pp. 1606-1615, 2004.

[24] F. Adam, A. Kauskot, J.-P. Rosa, and M. Bryckaert, "Mitogenactivated protein kinases in hemostasis and thrombosis," Journal of Thrombosis and Haemostasis, vol. 6, no. 12, pp. 2007-2016, 2008.

[25] M. Hayden, M. Pignone, C. Phillips, and C. Mulrow, "Aspirin for the primary prevention of cardiovascular events: a summary of the evidence for the U.S. Preventive Services Task Force," Annals of Internal Medicine, vol. 136, no. 2, pp. 161-172, 2002.

[26] T. A. Pearson, S. N. Blair, S. R. Daniels et al., "AHA Guidelines for Primary Prevention of Cardiovascular Disease and Stroke: 2002 Update: consensus panel guide to comprehensive risk reduction for adult patients without coronary or other atherosclerotic vascular diseases," Circulation, vol. 106, no. 3, pp. 388-391, 2002.

[27] R. S. Eidelman, P. R. Hebert, S. M. Weisman, and C. H. Hennekens, "An update on aspirin in the primary prevention of cardiovascular disease," Archives of Internal Medicine, vol. 163, no. 17, pp. 2006-2010, 2003.

[28] A. Panossian and G. Wikman, "Pharmacology of Schisandra chinensis Bail.: an overview of Russian research and uses in medicine," Journal of Ethnopharmacology, vol. 118, no. 2, pp. 183-212, 2008.

[29] R. Begum, M. Sharma, K. K. Pillai, V. Aeri, and M. A. Sheliya, "Inhibitory effect of Careya arborea on inflammatory biomarkers in carrageenan-induced inflammation," Pharmaceutical Biology, vol. 53, no. 3, pp. 437-445, 2015.

[30] A. G. Zaman, G. Helft, S. G. Worthley, and J. J. Badimon, "The role of plaque rupture and thrombosis in coronary artery disease," Atherosclerosis, vol. 149, no. 2, pp. 251-266, 2000.

[31] M. J. Berridge, M. D. Bootman, and H. L. Roderick, "Calcium signalling: dynamics, homeostasis and remodelling," Nature Reviews Molecular Cell Biology, vol. 4, no. 7, pp. 517-529, 2003.

[32] Z. Li, M. K. Delaney, K. A. O’Brien, and X. Du, "Signaling during platelet adhesion and activation," Arteriosclerosis, Thrombosis, and Vascular Biology, vol. 30, no. 12, pp. 2341-2349, 2010.

[33] Z. M. Ruggeri and G. L. Mendolicchio, "Adhesion mechanisms in platelet function," Circulation Research, vol. 100, no. 12, pp. 1673-1685, 2007.

[34] S. M. Schoenwaelder, A. Ono, W. S. Nesbitt, J. Lim, K. Jarman, and S. P. Jackson, "Phosphoinositide 3-kinase p110ßregulates integrin $\alpha \operatorname{IIb} \beta 3$ avidity and the cellular transmission of contractile forces," Journal of Biological Chemistry, vol. 285, no. 4, pp. 2886-2896, 2010.

[35] H. Wu, M. Ye, J. Yang et al., "Nicorandil protects the heart from ischemia/reperfusion injury by attenuating endoplasmic reticulum response-induced apoptosis through PI3K/Akt signaling pathway," Cellular Physiology and Biochemistry, vol. 35, no. 6, pp. 2320-2332, 2015.

[36] S. Wolfrum, A. Dendorfer, Y. Rikitake et al., "Inhibition of Rho-kinase leads to rapid activation of phosphatidylinositol 3-kinase/protein kinase Akt and cardiovascular protection," Arteriosclerosis, Thrombosis, and Vascular Biology, vol. 24, no. 10, pp. 1842-1847, 2004.

[37] S. Roger, M. Pawlowski, A. Habib, M. Jandrot-Perrus, J.-P. Rosa, and M. Bryckaert, "Costimulation of the Gi-coupled $\mathrm{ADP}$ receptor and the Gq-coupled $\mathrm{TXA}_{2}$ receptor is required for ERK2 activation in collagen-induced platelet aggregation," FEBS Letters, vol. 556, no. 1-3, pp. 227-235, 2004. 


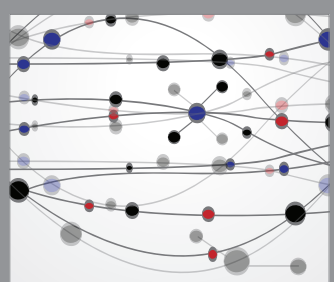

The Scientific World Journal
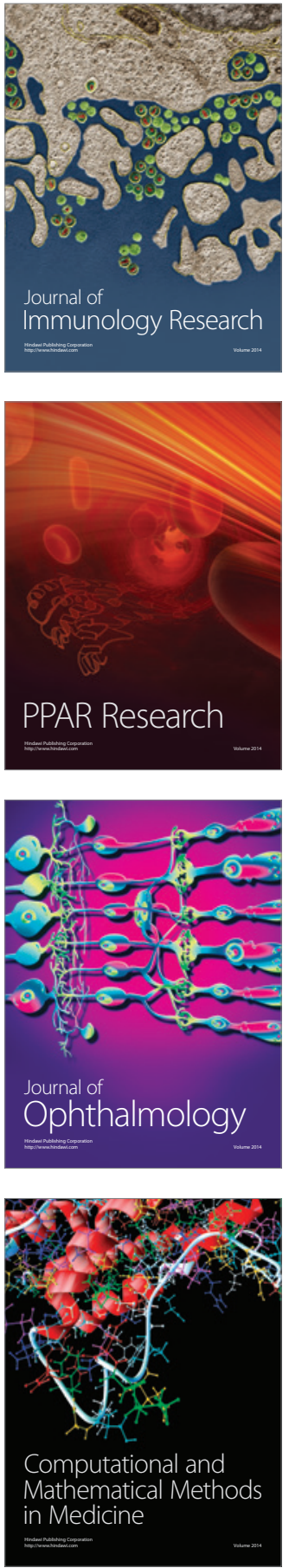

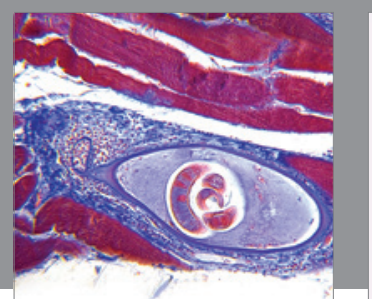

Gastroenterology Research and Practice
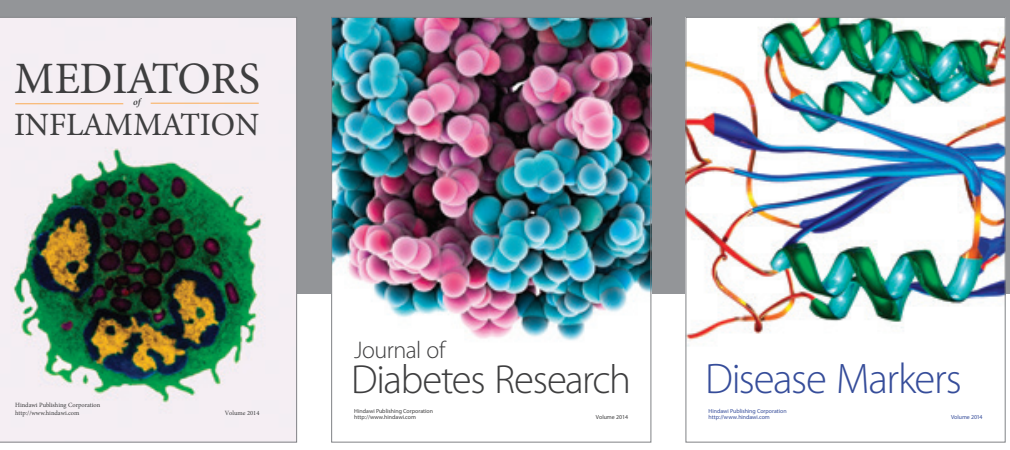

Disease Markers

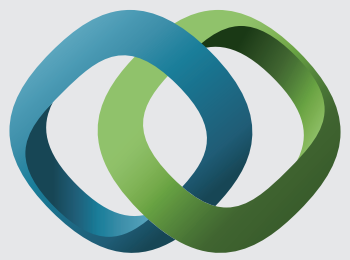

\section{Hindawi}

Submit your manuscripts at

https://www.hindawi.com
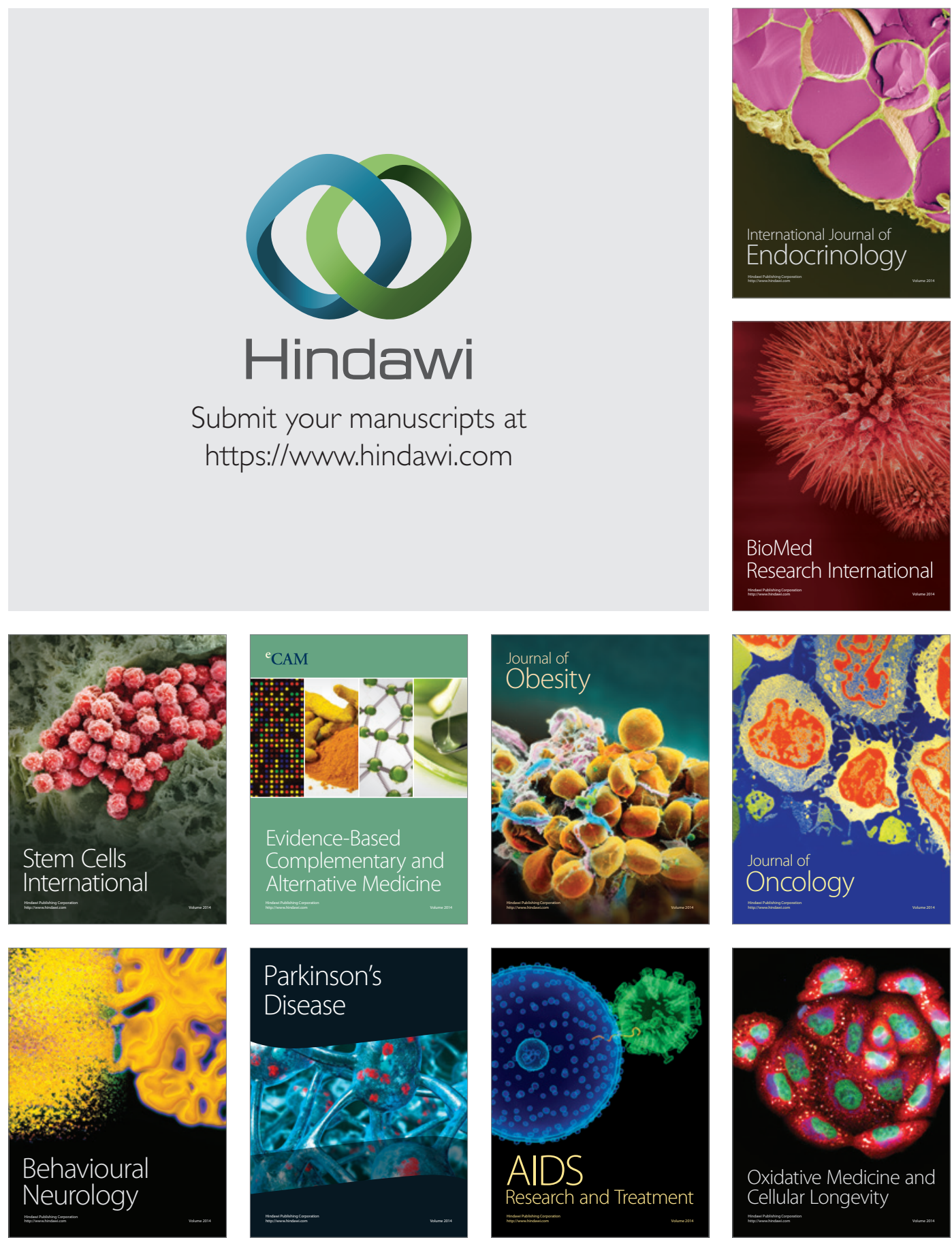